\section{Prognostische Mutationen bei MDS nach alloSCT}

Die einzige kurative Therapie myelodysplastischer Syndrome (MDS) ist die allogene Stammzelltransplantation (alloSCT). Die Mortalität durch Rezidive oder therapiebedingte Nebenwirkungen ist immer noch hoch. Mutationen könnten eine Differenzialtherapie für bestimmte Subgruppen ermöglichen.

D e meisten prognostischen Scores für MDS beziehen sich bislang auf klinische Eigenschaften und weniger auf Mutationen, die den Verlauf der Erkrankung entscheidend mitbestimmen. Deshalb wurde bei 1.520 Patienten, die zwischen 2005-2014 wegen eines MDS eine alloSCT erhalten und keine akute Leukämie ( $\geq 20 \%$ Blasten), myelomonozytäre Leukämie oder Überlappungssyndrome mit myeloproliferativen Neoplasien hatten, nach Mutationen gefahndet.

Bei $79 \%$ der Patienten wurden mindestens 1, median 2 Treibermutationen nachgewiesen (Bereich 0-15). Zahlenmäßig am häufigsten waren ASXL-1TP53- und DNMT3A-Mutationen mit mehr als $15 \%$. In der multivariaten Ana- lyse waren lediglich TP53-Mutationen mit einem verkürzten Überleben assoziiert (HR 1,71; $<<0,001)$. Mit einer kürzeren Zeit bis zum Rezidiv gingen TP53Mutationen (HR 2,03; p < 0,001) und Mutationen im RAS-Pathway (NRAS, KRAS, NFL, FLT3, Kit \& andere; HR $1,56 ; p=0,002)$ einher. Die JAK2-V617FMutationen waren mit einer höheren Todesrate ohne Rezidiv verknüpft (HR 2,1; $\mathrm{p}<0,001)$.

Erneut wurde die Bedeutung nichtgenetischer Faktoren bestätigt.

Während bei der TP53-Mutation das Gesamtüberleben unter jungen und älteren Patienten ähnlich war (HR 0,86; p $=0,50)$, überlebten Patienten ohne TP53Mutation im Alter $<40$ Jahre signifikant länger als ältere (HR 0,54; $\mathrm{p}<0,001)$.
Fehlen zusätzliche klinische Risikofaktoren (etwa $<30.000$ Thrombozyten zum Zeitpunkt der Transplantation oder Knochenmarkblasten $\geq 15 \%$ bei Diagnose), haben junge Patienten mit klassischem MDS ohne TP53-Mutation eine günstige Prognose: 3 Jahre nach HSCT überlebten $82 \%$.

Die Effekte der TP53-Mutation waren unabhängig von der Art der Konditionierung, myeloablativ oder dosisreduziert. Dagegen gab es frühe Rezidive bei Patienten mit Mutation im RAS-Pathway nur nach dosisreduzierter Konditionierung.

Fazit: Die Bestimmung des Mutationsstatus bei MDS-Patienten ermöglicht zusätzlich zu klinischen Risikokriterien die Definition von Risikogruppen. Der Mutationsstatus von TP53 ist von besonderer Bedeutung und kann künftig die Therapieplanung beeinflussen.

Brigitte Schalhorn

Lindsley RC et al. Prognostic mutations in myelodysplastic syndrome after stem-cell transplantation. N Engl J Med. 2017;376(6):536-47.

\section{BTK-Hemmung beim Marginalzonen-Lymphom}

Das Marginalzonen-Lymphom (MZL) ist ein heterogenes B-Zell-Lymphom, für das es bislang keine Standardtherapie gibt. Es ist häufig im Zusammenhang mit chronischen Infektionen wie Hepatitis $C$ oder Helicobacter pylori, die den B-Zell-Rezeptor(BCR)-Signalweg aktivieren und so eine aberrante B-Zell-Proliferation auslösen können. Nun wurde Ibrutinib bei MZL getestet, das die im BCR-Signalweg zentrale Bruton-Tyrosinkinase (BTK) hemmt.

$\mathrm{B}_{\mathrm{T}}^{\mathrm{e}}$ ei fortgeschrittenen MZL reicht das Therapiespektrum von einer Monotherapie mit einem Zytostatikum über eine Monotherapie mit einem monoklonalen CD20-Antikörper bis hin zu einer kombinierten Chemoimmuntherapie. In einer multizentrischen Phase-II-Studie wurden Wirksamkeit und Verträglichkeit einer Monotherapie mit Ibrutinib bei Patienten mit rezidiviertem oder refraktärem MZL und Vortherapie mit einem Anti-CD20-Antikörper geprüft. Die 63 Patienten erhielten $560 \mathrm{mg} / \mathrm{d}$ Ibrutinib bis zum Progress oder nicht akzeptabler Toxizität. Primärer Endpunkt war die Gesamtansprechrate (ORR). 32 Patienten
(51\%) hatten ein extranodales, $22 \%$ ein splenisches und $27 \%$ ein nodales MZL, 21 (57\%) einen Knochenmarkbefall. Sie hatten vorher median 2 systemische Therapien erhalten, $22 \%$ waren refraktär gegenüber ihrer letzten Therapie.

Bei den 60 auswertbaren Patienten betrug die ORR $48 \%$, sie erwies sich als über alle Tumorcharakteristika hinweg konsistent. Auffallend war die meist lange Ansprechdauer; nach median 19,4 Monaten war der Medianwert noch nicht erreicht. Das mediane progressionsfreie Überleben belief sich auf 14,2 Monate.

Die 3 häufigsten mit der Therapie verbundenen unerwünschten Ereignisse
(AE) vom Grad $\geq 3$ waren Fatigue ( $44 \%$ ), Diarrhö (43\%) und Anämie (33\%). Insgesamt wurde die Monotherapie mit Ibrutinib in dieser Studie vergleichsweise gut vertragen, und das AE-Profil stimmte mit dem aus anderen Ibrutinib-Studien bei Patienten mit Non-Hodgkin-Lymphomen oder chronischer lymphatischer Leukämie überein. Die gute Wirksamkeit von Ibrutinib beim MZL spiegelt sich vor allem in der hohen Rate klinischen Nutzens (definiert als stabile Erkrankung oder besser) von $83 \%$ und einer Reduktion der Tumorlast bei $78 \%$ wider. Dies deutet darauf hin, dass die BTK in der Pathophysiologie der MZL eine wichtige Rolle spielen könnte.

Fazit: Eine Monotherapie mit Ibrutinib induzierte anhaltende Remissionen (ORR $48 \%$ ) bei vorbehandelten Patienten mit MZL und zeigte ein günstiges Nutzen-Risiko-Profil. Brigitte Schalhorn

Noy A et al. Targeting Bruton tyrosine kinase with ibrutinib in relapsed/refractory marginal zone lymphoma. Blood. 2017;129(16):2224-32. 